\title{
Molecular technique identifies the pathogen responsible for culture negative infective endocarditis
}

\author{
G Y Shin, R J Manuel, S Ghori, S Brecker, A S Breathnach
}

Heart 2005;91:e47 (http://www.heartjnl.com/cgi/content/full/91/6/e47). doi: 10.1136/hrt.2004.056762

\begin{abstract}
A case of culture negative endocarditis complicated by immune complex glomerulonephritis and severe aortic regurgitation necessitated aortic valve replacement. Empirical treatment with penicillin and gentamicin according to UK guidelines was started. The pathogen, Streptococcus sanguis, was later identified by polymerase chain reaction amplification and sequencing of bacterial $16 \mathrm{~S}$ ribosomal RNA. This molecular technique is likely to be of increasing importance in determining the aetiology of culture negative infective endocarditis, thus providing essential treatment and epidemiological information.
\end{abstract}

$\mathrm{T}$ he diagnosis of infective endocarditis (IE) can remain problematic in certain cases. Traditionally, clinical suspicion, conventional microbiology techniques, and echocardiography lead to the diagnosis of IE. Many cases of IE remain culture negative when prior antibiotics have been given. 16S rRNA sequencing is a recent sensitive diagnostic tool that is increasingly being used to diagnose culture negative endocarditis.

\section{CASE REPORT}

A 62 year old previously fit and well man presented to hospital with a one month history of vomiting, weight loss, and a low grade fever. Amoxicillin-clavulanic acid and erythromycin were started on admission for a presumed chest infection. He was found to have acute renal failure. A renal biopsy was therefore taken, and histology showed a focal, segmental, proliferative, and necrotising glomerulonephritis with $19 \%$ crescents, secondary to immune complex deposition.

A transthoracic echocardiogram recorded a few days later showed notable thickening of the aortic valve leaflet tips with a small mobile mass on the right coronary cusp. Three sets of blood cultures taken at this time, 10 days after he had been started on antibiotics, were negative. Possible culture negative endocarditis was diagnosed by Duke's criteria ${ }^{2}$ based on a positive echocardiogram (major criterion) and immune complex glomerulonephritis and fever (both minor criteria). His antibiotics were changed to intravenous benzylpenicillin $2.4 \mathrm{~g}$ every four hours and gentamicin $120 \mathrm{mg}$ twice daily in accordance with UK guidelines for the empirical treatment of endocarditis, which assume streptococcal species to be the most likely organism in this context. ${ }^{3}$

Over the next two weeks, he made a good recovery. However, on day 13, his fever returned. He was also noted to have a vasculitic rash on his lower limbs and splinter haemorrhages on his metatarsals.

A transoesophageal echocardiogram showed thickening of the tips of the entire left coronary cusp of the aortic valve and the right coronary cusp tip adjacent to it, associated with a

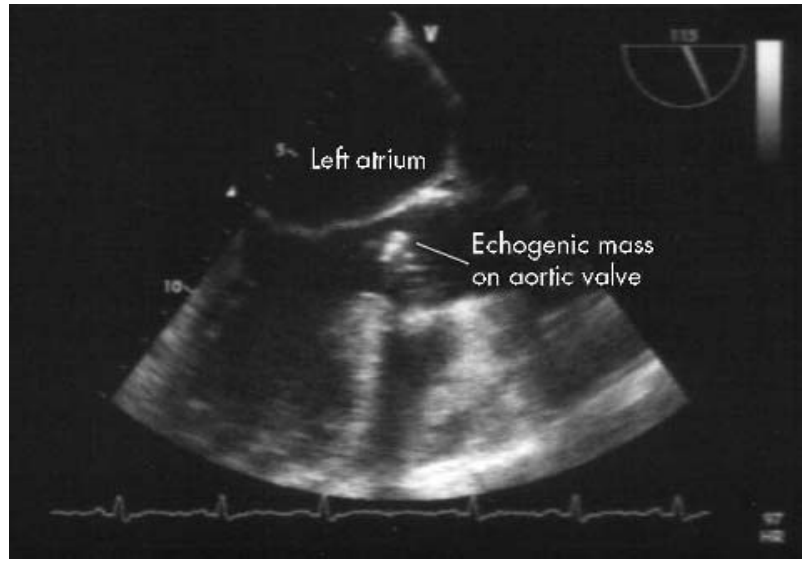

Figure 1 Transoesophageal echocardiogram showed thickening of the tips of the entire left coronary cusp of the aortic valve and the right coronary cusp tip adjacent to it, associated with a broad jet of severe aortic regurgitation.

broad jet of severe aortic regurgitation, requiring urgent valve replacement (fig 1).

The histological appearance of the aortic valve was consistent with subacute bacterial endocarditis. Numerous Gram positive cocci were visualised on microscopy. Culture of the valve was negative.

16S rRNA based sequencing on a portion of the valve tissue gave a 100\% identification for Streptococcus sanguis.

The patient made a good postoperative recovery and was discharged six weeks after surgery.

\section{DISCUSSION}

The diagnosis of IE remains challenging. Once the diagnosis has been considered, traditional clinical and laboratory methods are still the basic tools at the physician's disposal. Imaging the cardiac valves by echocardiography, particularly transoesophageal echocardiography, is helpful.

Most common IE pathogens are easily cultured in modern medical microbiology laboratories. The organism can usually be identified to species level and accurate antimicrobial sensitivities can be determined. However, in the UK, no organism is grown by conventional culture in around $10-15 \%$ of IE cases. ${ }^{1}$

The most common cause of culture negative IE is the use of antibiotics before blood cultures have been taken. ${ }^{4}$ Infection by rare or fastidious organisms is less common.

Culture negative IE caused by unusual organisms such as Bartonella, Brucella, and Legionella species can be diagnosed by the detection of a specific antibody response in the patient's serum. ${ }^{14}$ Other pathogens such as Coxiella burnetti, which is responsible for $\mathrm{Q}$ fever, can also be diagnosed by serology. $C$ burnetti is hazardous to laboratory staff (Advisory Committee on Dangerous Pathogens Hazard Group 3 pathogen ${ }^{5}{ }^{6}$ ) and is 
difficult to grow, which is why serology is the principal method by which this organism is diagnosed. In the case of $C$ burnetti, serology is also used to monitor response to treatment, which is difficult and often requires surgery. Growth of fastidious bacteria, such as the HACEK group (Haemophilus parainfluenzae, Haemophilus aphrophilus, Actinobacillus actinomycetemcomitans, Cardiobacterium hominis, Eikenella species, and Kingella species), requires prolonged incubation of blood cultures.

Molecular techniques such as polymerase chain reaction (PCR) of the bacterial 16S rRNA gene can establish the aetiology of culture negative IE when traditional methods fail, ${ }^{1}$ as in this case. There are four main scenarios when 16S rRNA PCR is useful in diagnosing the pathogen responsible for a case of culture negative IE. Firstly, in cases where no cultures have been taken before the initiation of antibiotic, 16S rRNA may be the only way the aetiology can be determined. The second scenario is when the pathogen responsible is difficult to grow by traditional microbiology laboratory methods. Thirdly, some pathogens, such as $C$ burnetti and Brucella species, may pose a significant biohazard to laboratory staff if grown in the laboratory. Lastly, rare bacteria such as Tropheryma whippelii and Bartonella species are being identified by 16S rRNA PCR followed by phylogenetic analysis in cases of culture negative IE that previously would never have had a pathogen identified.

This case serves as a reminder that most cases of culture negative endocarditis are caused by streptococci likely to be sensitive to penicillin, even when blood cultures are negative because of prior antibiotic use.

Molecular diagnostic techniques have the potential to identify the infective agent causing many cases of blood culture negative IE, thus providing useful clinical and epidemiological information. ${ }^{7}$
Authors' affiliations

G Y Shin, R J Manuel, S Ghori, A S Breathnach, Department of Medical Microbiology, St George's Hospital Medical School, London, UK S Brecker, Department of Cardiology, St George's Hospital Medical School

Source(s) of support (grants, equipment, drugs): none

No competing interests to declare.

Patient consent: We confirm that we have received written patient consent on the PMJ patient consent pro forma. This consent is dated 14 October 2004.

Correspondence to: Dr Gee Yen Shin, Department of Medical Microbiology, St George's Hospital, Blackshaw Road, London SW17 OQT, UK; gshin@sghms.ac.uk

Accepted 29 November 2004

\section{REFERENCES}

1 Millar BC, Altwegg M, Raoult $D$, et al. Culture-negative endocarditis: causes, diagnosis and treatment. Rev Med Microbiol 2000;1 1:59-75.

2 Durack DT, Lukes AS, Bright DK, et al. New criteria for diagnosis of infective endocarditis: utilization of specific echocardiographic findings. Am J Med 1994;96:200-8.

3 Simmons NA, Ball AP, Eykyn SJ, for the Working Party of the British Society for Antimicrobial Chemotherapy, et al. Antibiotic treatment of streptococcal, enterococcal, and staphylococcal endocarditis. Heart streptococcal, enteroc

4 Bayer AS, Bolger AF, Taubert KA, et al. Diagnosis and management of infective endocarditis and its complications. Circulation 1998;98:2936-48.

5 Advisory Committee on Dangerous Pathogens. Categorisation of biological agents according to hazard and categories of containment, 4th ed. Sudbury, Suffolk: HSE Books, 1995

6 Advisory Committee on Dangerous Pathogens. Supplement to categorisation of biological agents according to hazard and categories of containment. Sudbury, Suffolk: HSE Books, 1998.

7 Millar B, Moore J, Mallon P, et al. Molecular diagnosis of infective endocarditis: a new Duke's criterion. Scand J Infect Dis 2001;33:673-80. 